\title{
THE ONSET OF CONVECTION IN A ROTATING MULTICOMPONENT FLUID LAYER
}

\author{
Jyoti Prakash, Virender Singh, Rajeev Kumar, Kultaran Kumari \\ Department of Mathematics and Statistics, Himachal Pradesh University, Shimla, India \\ e-mail: jpsmaths67@gmail.com
}

\begin{abstract}
The onset of convective instability is analysed in a rotating multicomponent fluid layer in which density depends on $n$ stratifying agents (one of them is heat) having different diffusivities. Two problems have been analysed mathematically. In the first problem, a sufficient condition is derived for the validity of the principle of the exchange of stabilities. Further, when the complement of this condition holds good, oscillatory motions of neutral or growing amplitude can exist, and thus it is important to derive upper bounds for the complex growth rate of such motions when at least one of the bounding surfaces is rigid so that exact solutions of the problem in closed form are not obtainable. Thus, as the second problem, bounds for the complex growth rates are also obtained. Above results are uniformly valid for quite general nature of the bounding surfaces.
\end{abstract}

Keywords: multicomponent convection, principle of exchange of stabilities, oscillatory motions, complex growth rate, concentration Rayleigh number, Lewis number

\section{Introduction}

When density of a fluid is determined by two stratifying agents, such as heat and salt diffusing at different rates, the fluid at rest can be unstable even if its density increases downward. This convective phenomenon is known as thermosolutal convection or more generally as double diffusive convection. This phenomenon has, now, been extensively studied. For review on the subject of double diffusive convection one may be referred to (Turner, 1973, 1974, 1985; Brandt and Fernando, 1996; Radko, 2013; Sekar et al., 2013).

Although the subject of double diffusive convection is still an important area of research (Sekar et al., 2013; Kellner and Tilgner, 2014; Nield and Kuznetsov, 2011; Schmitt, 2011), there are many fluid systems where more than two components are present (Turner, 1985; Griffiths, 1979b). Examples of such systems include the solidification of molten alloys, Earth core, geothermally heated lakes, sea water, magmas and their laboratory models. The presence of more than one salt in fluid mixtures is very often requested for describing natural phenomena such as contaminant transport, acid rain effects, underground water flow and warming of the stratosphere. The subject of more than two stratifying agents has attracted many researchers (Griffiths, 1979a,b; Pearlstein et al., 1989; Rionero, 2013a,b, 2014; Lopez et al., 1990; Terrones, 1993; Poulikakos, 1985; Shivakumara and Naveen Kumar, 2014). In double diffusive convection (Turner, 1974) or, more generally, in multicomponent convection (Turner, 1985; Griffiths, 1979a) instability may occur in two kinds: first in form of steady (or stationary) convection which is called as 'salt finger' modes and the second in form of oscillatory motions of growing amplitude (or overstability) which is called as 'diffusive convection'. When a warm and saltier fluid lies above a cold and fresh fluid then stationary convection is preferred, and when a cold and fresher fluid lies above a warm and saltier fluid then oscillatory motions are preferred. The essence of these researchers is that small concentrations of the third diffusing component with a smaller mass 
diffusivity can have a significant effect upon the nature of diffusive instabilities and diffusive convection. The salt finger modes are simultaneously unstable under a wide range of conditions when the density gradients due to components with the greatest and smallest diffusivity are of the same signs even if the overall density stratification is hydrostatically stable. These researchers also notice some fundamental differences between doubly and triply diffusive convection. One is that if the gradients of two of the stratifying agents are held fixed, then three critical values of the Rayleigh number of the third agent are sometimes required to specify the linear stability criteria (in double diffusive convection only one critical Rayleigh number is required). The other difference is that the onset of convection for the case of free boundaries may occur via quassiperiodic bifurcation from the motionless basic state.

Now the triply diffusive convection despite its complexities has also been well studied. But, to the author knowledge, not many investigations have been conducted on stability theory when more than three components are present, which may be, perhaps, due to the complexities involved in mathematical calculations and numerical computations. Some worth researches which may be referred here are due to Terrones and Pearlstein (1989) who derived analytical results for $n$ components and numerical results for $n=5$ using dynamically free boundary conditions. Later Lopez et al. (1990) predicted that the results of triply diffusive convection may be extended to multicomponent convection with $n$ components for rigid surfaces also. Further significant contributions to multicomponent convection are due to Ryzhkov and Shevtsova (2007, 2009) and Ryzhkov (2013).

The establishment of nonoccurrence of any slow oscillatory motions which may be neutral or unstable implies the validity of the principle of the exchange of stabilities (PES). The validity of this principle in stability problems eliminates unsteady terms from linearized perturbation equations which results in notable mathematical simplicity since the transition from stability to instability occurs via a marginal state which is characterized by the vanishing of both real and imaginary parts of the complex time eigenvalue associated with the perturbation. Pellew and Southwell (1940) proved the validity of PES (i.e. occurrence of stationary convection) for the classical Rayleigh-Benard instability problem. Prakash et al. (2014a) established such a criterion for the triply diffusive convection problem.

To study the effect of rotation on a multicomponent fluid layer is an interesting topic. Prakash et al. (2014b) derived a sufficient condition for the occurrence of stationary convection and upper bounds (Prakash et al., 2015) for the complex growth rate of an arbitrary oscillatory motion of neutral or growing amplitude in rotatory hydrodynamic triply diffusive convection. The further extension of these results to the problem of the onset of convection in a multicomponent fluid layer in the domains of astrophysics and terrestrial physics, wherein the liquid concerned has the property of electrical conduction and the magnetic field and rotation are prevalent, is very much sought after in the present context.

In the present work, we analyse the onset of buoyancy driven convection in a multicomponent fluid layer in the presence of uniform vertical rotation. We generalize the existing results of the rotatory hydrodynamic triply diffusive convection problem concerning the validity of the principle of the exchange of stabilities (Prakash et al., 2014b) and arresting the complex growth rate of oscillatory motion (when it occurs) (Prakash et al., 2015) which are important especially when at least one boundary is rigid so that exact solutions in the closed form are not obtainable. To the authors knowledge, no such results have been obtained so far for the hydrodynamical systems with more than three components. The results derived herein are uniformly valid for any combination of the rigid and free boundaries and the results of doubly diffusive (Banerjee et al., 1981; Gupta et al., 1986) and triply diffusive convection (Prakash et al., 2014a,b,c, 2015) follow as a consequence. Further, the importance of the results obtained herein lies in that these results may be used for any rotatory hydrodynamic multicomponent system where no mathematical calculation or numerical computation is possible. 


\section{Mathematical formulation and analysis}

A viscous finitely heat conducting Boussinesq fluid of infinite horizontal extension is statistically confined between two horizontal boundaries $z=0$ and $z=d$ which are respectively maintained at uniform temperatures $T_{0}$ and $T_{1}\left(<T_{0}\right)$ and uniform concentrations $S_{10}, S_{20}, \ldots, S_{(n-1) 0}$ and $S_{11}\left(<S_{10}\right), S_{21}\left(<S_{20}\right), \ldots, S_{(n-1) 1}\left(<S_{(n-1) 0}\right)$ in the force field of gravity and in the presence of uniform vertical rotation (as shown in Fig. 1). It is assumed that the cross-diffusion effects of the stratifying agents can be neglected.

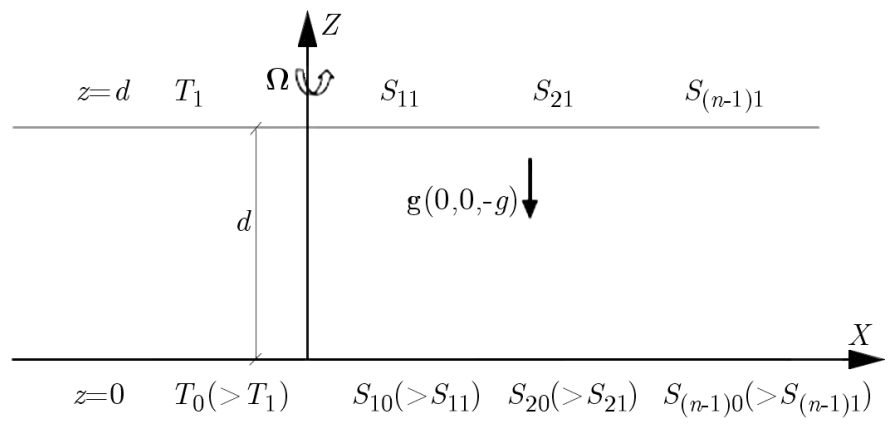

Fig. 1. Physical configuration

The basic equations that govern the motion of the rotatory hydrodynamic multicomponent fluid layer are as follows (Prakash et al., 2014b; Terrones and Pearlstein, 1989)

$$
\begin{aligned}
& \frac{\partial u_{j}}{\partial x_{j}}=0 \\
& \frac{\partial u_{i}}{\partial t}+u_{j} \frac{\partial u_{i}}{\partial x_{j}}=-\frac{\partial}{\partial x_{i}}\left(\frac{P_{1}}{\rho_{0}}-\frac{1}{2} \mid\left(\boldsymbol{\Omega} \times\left.\mathbf{r}\right|^{2}\right)+\left(1+\frac{\delta \rho}{\rho_{0}}+\frac{\delta \rho_{1}}{\rho_{0}}+\frac{\delta \rho_{2}}{\rho_{0}}+\ldots+\frac{\delta \rho_{n-1}}{\rho_{0}}\right) X_{i}\right. \\
& +2 \varepsilon_{i j k} u_{j} \Omega_{k}+\nu \nabla^{2} u_{i} \\
& \frac{\partial T}{\partial t}+u_{j} \frac{\partial T}{\partial x_{j}}=\kappa \nabla^{2} T \\
& \frac{\partial S_{1}}{\partial t}+u_{j} \frac{\partial S_{1}}{\partial x_{j}}=\kappa_{1} \nabla^{2} S_{1} \\
& \frac{\partial S_{2}}{\partial t}+u_{j} \frac{\partial S_{2}}{\partial x_{j}}=\kappa_{2} \nabla^{2} S_{2} \\
& \frac{\partial S_{n-1}}{\partial t}+u_{j} \frac{\partial S_{n-1}}{\partial x_{j}}=\kappa_{n-1} \nabla^{2} S_{n-1}
\end{aligned}
$$

where $\rho$ is density; $t$ is time; $x_{j}(j=1,2,3)$ are cartesian coordinates $x, y, z ; u_{j}(j=1,2,3)$ are velocity components; $X_{i}(i=1,2,3)$ are components of the external force in the $x, y, z$ directions, respectively; $P_{1}$ is pressure; $\mu$ is viscosity; $\boldsymbol{\Omega}$ is angular velocity; $T$ is temperature, $\kappa$ is the coefficient of thermal diffusivity; $S_{1}, S_{2}, \ldots, S_{n-1}$ are $n-1$ concentrations and $\kappa_{1}, \kappa_{2}, \ldots, \kappa_{n-1}$ are respectively the coefficients of mass diffusivity of $S_{1}, S_{2}, \ldots, S_{n-1}$ with $\kappa_{1}>\kappa_{2}>\ldots>\kappa_{n-1}$.

The above basic equations must be supplemented by the equation of state

$$
\rho=\rho_{0}\left[1+\alpha\left(T_{0}-T_{1}\right)-\alpha_{1}\left(S_{10}-S_{11}\right)-\alpha_{2}\left(S_{20}-S_{21}\right)-\ldots-\alpha_{n-1}\left(S_{(n-1) 0}-S_{(n-1) 1}\right)\right]
$$

where $\alpha, \alpha_{1}, \alpha_{2}, \ldots, \alpha_{n-1}$ are respectively the coefficients of volume expansion due to temperature variation and concentration variations for $n-1$ concentration components $S_{1}, S_{2}, \ldots, S_{n-1}$. 
$\rho_{0}$ is the value of $\rho$ at $z=0 . \nu=\mu / \rho_{0}$ is kinematic viscosity and $\frac{P_{1}}{\rho_{0}}-\frac{1}{2}|\boldsymbol{\Omega} \times \mathbf{r}|^{2}$ is hydrostatic pressure.

The basic state is assumed to be stationary, and the standard linear stability analysis procedure as outlined in the studies of Prakash et al. (2014b) is followed to obtain the following non-dimensional stability equations

$$
\begin{aligned}
& \left(D^{2}-a^{2}\right)\left(D^{2}-a^{2}-\frac{\sigma}{\operatorname{Pr}}\right) w=\operatorname{Ra} a^{2} \theta-R_{1} a^{2} \phi_{1}-R_{2} a^{2} \phi_{2}-\ldots-R_{n-1} a^{2} \phi_{n-1}+\operatorname{Ta} D \zeta \\
& \left(D^{2}-a^{2}-\sigma\right) \theta=-w \\
& \left(D^{2}-a^{2}-\frac{\sigma}{\mathrm{Le} 1}\right) \phi_{1}=-\frac{w}{\mathrm{Le}_{1}} \\
& \left(D^{2}-a^{2}-\frac{\sigma}{\mathrm{Le}_{2}}\right) \phi_{2}=-\frac{w}{\mathrm{Le}_{2}} \\
& \vdots \\
& \left(D^{2}-a^{2}-\frac{\sigma}{\mathrm{Le}_{n-1}}\right) \phi_{n-1}=-\frac{w}{\mathrm{Le}_{n-1}}
\end{aligned}
$$

and

$$
\left(D^{2}-a^{2}-\frac{\sigma}{\operatorname{Pr}}\right) \zeta=-D w
$$

respectively.

Equations (2.3) and (2.4) are to be solved using the following appropriate boundary conditions:

$-w=0=\theta=\phi_{1}=\phi_{2}=\ldots=\phi_{n-1}$ on both the horizontal boundaries which are at

$$
z=0 \quad \text { and } \quad z=1
$$

— and on rigid boundary

$$
D w=\zeta=0
$$

— or on free boundary

$$
D^{2} w=D \zeta=0
$$

the meaning of the symbols involved in Eqs. (2.3) and (2.4) from the physical point of view are as follows: $z$ is the vertical coordinate, $D=d / d z$ is differentiation w.r.t. $z, a^{2}>0$ is square of the wave number, $\operatorname{Pr}>0$ is the thermal Prandtl number which is a measure of relative importance of heat conduction and viscosity of the fluid and varies from fluid to fluid. For air $\operatorname{Pr}=0.7$ (approximately), for water $\operatorname{Pr}=7$ (approximately), for mercury $\operatorname{Pr}=0.044$ (approximately) and for glycerine $\operatorname{Pr}=7250$. The Prandtl number of some fluids (particularly water) depends considerably on temperature. $\mathrm{Le}_{1}>0, \mathrm{Le}_{2}>0, \ldots, \mathrm{Le}_{n-1}>0$ are the Lewis numbers for $n-1$ concentrations $S_{1}, S_{2}, \ldots, S_{n-1}$, respectively, Ta $>0$ is the Taylor number, Ra $>0$ is the thermal Rayleigh number, $R_{1}>0, R_{2}>0, \ldots, R_{n-1}>0$ are the concentration Rayleigh numbers for the $n-1$ concentration components. A concentration Rayleigh number is the ratio of the buoyancy forces (which drive free convective transport of solute) to dispersive/viscous forces (which disperse solute and dissipate free convective transport). In the present problem, these have stabilizing effect on the onset of instability. $w$ is vertical velocity, $\theta$ is temperature and $\phi_{1}, \phi_{2}, \ldots, \phi_{n-1}$ are respective concentrations of the $n-1$ components. $\sigma=\sigma_{r}+\mathrm{i} \sigma_{i}$ is the complex growth rate where $\sigma_{r}$ and $\sigma_{i}$ are real constants. For $\sigma_{r}<0$, the system is always stable while for $\sigma_{r}>0$, the system becomes unstable. When $\sigma=0$, the system is marginally stable 
ensuring the validity of the principle of the exchange of stabilities. When $\sigma_{r} \geqslant 0$ and $\sigma_{i} \neq 0$, the overstability of periodic motion is possible and oscillatory motions of growing or neutral amplitude occur. It may further be noted that equations (2.3) and (2.4) describe an eigenvalue problem for $p$ and govern rotatory hydrodynamic multicomponent convection for quite general nature of the bounding surfaces.

Theorem 1: If $\left(w, \theta, \phi_{1}, \phi_{2}, \ldots, \phi_{n-1}, \zeta, \sigma\right)$, Ra $>0, R_{1}>0, R_{2}>0, \ldots, R_{n-1}>0$, Ta $>0$, $\sigma_{r} \geqslant 0$ is a solution to Eqs. (2.3) and (2.4) together with boundary conditions (2.5)-(2.7) and

$$
\frac{R_{1} \mathrm{Pr}}{2 \mathrm{Le}_{1}^{2} \pi^{4}}+\frac{R_{2} \mathrm{Pr}}{2 \mathrm{Le}_{2}^{2} \pi^{4}}+\ldots+\frac{R_{n-1} \mathrm{Pr}}{2 \mathrm{Le}_{n-1}^{2} \pi^{4}}+\frac{\mathrm{Ta}}{\pi^{4}} \leqslant 1
$$

then $\sigma_{i}=0$. In particular,

$$
\sigma_{r}=0 \Rightarrow \sigma_{i}=0 \quad \text { if } \quad \frac{R_{1} \operatorname{Pr}}{2 \mathrm{Le}_{1}^{2} \pi^{4}}+\frac{R_{2} \mathrm{Pr}}{2 \mathrm{Le}_{2}^{2} \pi^{4}}+\ldots+\frac{R_{n-1} \mathrm{Pr}}{2 \mathrm{Le}_{n-1}^{2} \pi^{4}}+\frac{\mathrm{Ta}}{\pi^{4}} \leqslant 1
$$

Proof: Multiplying Eq. (2.3) ${ }_{1}$ by $w^{*}$ (the superscript $*$ henceforth denotes complex conjugation) throughout and integrating the resulting equation over the vertical range of $z$, we have

$$
\begin{gathered}
\int_{0}^{1} w^{*}\left(D^{2}-a^{2}\right)\left(D^{2}-a^{2}-\frac{\sigma}{\operatorname{Pr}}\right) w d z=\operatorname{Ra} a^{2} \int_{0}^{1} w^{*} \theta d z-R_{1} a^{2} \int_{0}^{1} w^{*} \phi_{1} d z \\
-R_{2} a^{2} \int_{0}^{1} w^{*} \phi_{2} d z-\ldots-R_{n-1} a^{2} \int_{0}^{1} w^{*} \phi_{n-1} d z+\operatorname{Ta} \int_{0}^{1} w^{*} D \zeta d z
\end{gathered}
$$

Making use of Eqs. $(2.3)_{2-6}$ and (2.4), we can write

$$
\begin{aligned}
& \int_{0}^{1} w^{*}\left(D^{2}-a^{2}\right)\left(D^{2}-a^{2}-\frac{\sigma}{\operatorname{Pr}}\right) w d z=-\operatorname{Ra}^{2} \int_{0}^{1} \theta\left(D^{2}-a^{2}-\sigma^{*}\right) \theta^{*} d z \\
& \quad+R_{1} a^{2} \operatorname{Le}_{1} \int_{0}^{1} \phi_{1}\left(D^{2}-a^{2}-\frac{\sigma^{*}}{\mathrm{Le}}\right) \phi_{1}^{*} d z+R_{2} a^{2} \operatorname{Le}_{2} \int_{0}^{1} \phi_{2}\left(D^{2}-a^{2}-\frac{\sigma^{*}}{\mathrm{Le}_{2}}\right) \phi_{2}^{*} d z+\ldots \\
& \quad+R_{n-1} a^{2} \operatorname{Le}_{n-1} \int_{0}^{1} \phi_{n-1}\left(D^{2}-a^{2}-\frac{\sigma^{*}}{\mathrm{Le}_{n-1}}\right) \phi_{n-1}^{*} d z+\mathrm{Ta} \int_{0}^{1} \zeta\left(D^{2}-a^{2}-\frac{\sigma^{*}}{\operatorname{Pr}}\right) \zeta^{*} d z
\end{aligned}
$$

Integrating the various terms of Eq. (2.9) by parts for an appropriate number of times and utilizing boundary conditions (2.5)-(2.7), we obtain

$$
\begin{aligned}
& \int_{0}^{1}\left(\left|D^{2} w\right|^{2}+2 a^{2}|D w|^{2}+a^{4}|w|^{2}\right)+\frac{\sigma}{\operatorname{Pr}} \int_{0}^{1}\left(|D w|^{2}+a^{2}|w|^{2}\right) d z \\
& \quad=\operatorname{Ra}^{2} \int_{0}^{1}\left(|D \theta|^{2}+a^{2}|\theta|^{2}+\sigma^{*}|\theta|^{2}\right) d z-R_{1} a^{2} \operatorname{Le}_{1} \int_{0}^{1}\left(\left|D \phi_{1}\right|^{2}+a^{2}\left|\phi_{1}\right|^{2}+\frac{\sigma^{*}}{\mathrm{Le}_{1}}\left|\phi_{1}\right|^{2}\right) d z \\
& \quad-R_{2} a^{2} \operatorname{Le}_{2} \int_{0}^{1}\left(\left|D \phi_{2}\right|^{2}+a^{2}\left|\phi_{2}\right|^{2}+\frac{\sigma^{*}}{\mathrm{Le}_{2}}\left|\phi_{2}\right|^{2}\right) d z-\ldots
\end{aligned}
$$




$$
\begin{aligned}
& -R_{n-1} a^{2} \operatorname{Le}_{n-1} \int_{0}^{1}\left(\left|D \phi_{n-1}\right|^{2}+a^{2}\left|\phi_{n-1}\right|^{2}+\frac{\sigma^{*}}{\operatorname{Le}_{n-1}}\left|\phi_{n-1}\right|^{2}\right) d z \\
& -\operatorname{Ta} \int_{0}^{1}\left(|D \zeta|^{2}+a^{2}|\zeta|^{2}+\frac{\sigma^{*}}{\operatorname{Pr}}|\zeta|^{2}\right) d z
\end{aligned}
$$

Equating the imaginary parts of both sides of Eq. (2.10) and cancelling $\sigma_{i}(\neq 0)$ throughout from the resulting equation, we have

$$
\begin{aligned}
& \frac{1}{\operatorname{Pr}} \int_{0}^{1}\left(|D w|^{2}+a^{2}|w|^{2}\right) d z=-\operatorname{Ra}^{2} \int_{0}^{1}|\theta|^{2} d z+R_{1} a^{2} \int_{0}^{1}\left|\phi_{1}\right|^{2} d z \\
& \quad+R_{2} a^{2} \int_{0}^{1}\left|\phi_{2}\right|^{2} d z+\ldots+R_{n-1} a^{2} \int_{0}^{1}\left|\phi_{n-1}\right|^{2} d z+\frac{\operatorname{Ta}}{\operatorname{Pr}} \int_{0}^{1}|\zeta|^{2} d z
\end{aligned}
$$

Now, from equation $(2.3)_{3}$ we derive that

$$
\int_{0}^{1}\left(D^{2}-a^{2}-\frac{\sigma}{\mathrm{Le}_{1}}\right) \phi_{1}\left(D^{2}-a^{2}-\frac{\sigma^{*}}{\mathrm{Le}_{1}}\right) \phi_{1}^{*} d z=\frac{1}{\mathrm{Le}_{1}^{2}} \int_{0}^{1}|w|^{2} d z
$$

Integrating the various terms on the left hand side of Eq. (2.12) by parts for an appropriate number of times and making use of the boundary conditions on $\phi_{1}$, it follows that

$$
\begin{aligned}
& \int_{0}^{1}\left(\left|D^{2} \phi_{1}\right|^{2}+2 a^{2}\left|D \phi_{1}\right|^{2}+a^{4}\left|\phi_{1}\right|^{2}\right) d z+\frac{2 \sigma_{r}}{\mathrm{Le}_{1}} \int_{0}^{1}\left(\left|D \phi_{1}\right|^{2}+a^{2}\left|\phi_{1}\right|^{2}\right) d z \\
& \quad+\frac{|\sigma|^{2}}{\operatorname{Le}_{1}^{2}} \int_{0}^{1}\left|\phi_{1}\right|^{2} d z=\frac{1}{\operatorname{Le}_{1}^{2}} \int_{0}^{1}|w|^{2} d z
\end{aligned}
$$

Since $\sigma_{r} \geqslant 0$, it follows from Eq. (2.13) that

$$
2 a^{2} \int_{0}^{1}\left|D \phi_{1}\right|^{2} d z \leqslant \frac{1}{\operatorname{Le}_{1}^{2}} \int_{0}^{1}|w|^{2} d z
$$

Now, since $\phi_{1}, \phi_{2}, \ldots, \phi_{n-1}$ and $w$ satisfy the boundary conditions $\phi_{1}(0)=0=\phi_{1}(1)$, $\phi_{2}(0)=0=\phi_{2}(1), \ldots, \phi_{n-1}(0)=0=\phi_{n-1}(1), w(0)=0=w(1)$, we have from the Rayleigh-Ritz inequality (Schultz, 1973)

$$
\begin{aligned}
& \int_{0}^{1}\left|D \phi_{1}\right|^{2} d z \geqslant \pi^{2} \int_{0}^{1}\left|\phi_{1}\right|^{2} d z \\
& \int_{0}^{1}\left|D \phi_{2}\right|^{2} d z \geqslant \pi^{2} \int_{0}^{1}\left|\phi_{2}\right|^{2} d z \\
& \vdots \\
& \int_{0}^{1}\left|D \phi_{n-1}\right|^{2} d z \geqslant \pi^{2} \int_{0}^{1}\left|\phi_{n-1}\right|^{2} d z
\end{aligned}
$$


and

$$
\int_{0}^{1}|D w|^{2} d z \geqslant \pi^{2} \int_{0}^{1}|w|^{2} d z
$$

respectively.

Utilizing inequalities $(2.15)_{1}$ and (2.16) in inequality (2.14), we get

$$
a^{2} \int_{0}^{1}\left|\phi_{1}\right|^{2} d z \leqslant \frac{1}{2 \operatorname{Le}_{1}^{2} \pi^{4}} \int_{0}^{1}|D w|^{2} d z
$$

In the same manner, we obtain from Eqs. $(2.3)_{4-6}$ the inequalities

$$
\begin{aligned}
& a^{2} \int_{0}^{1}\left|\phi_{2}\right|^{2} d z \leqslant \frac{1}{2 \operatorname{Le}_{2}^{2} \pi^{4}} \int_{0}^{1}|D w|^{2} d z \\
& \vdots \\
& a^{2} \int_{0}^{1}\left|\phi_{n-1}\right|^{2} d z \leqslant \frac{1}{2 \operatorname{Le}_{n-1}^{2} \pi^{4}} \int_{0}^{1}|D w|^{2} d z
\end{aligned}
$$

respectively.

Now for the case of rigid boundaries, $\zeta(0)=0=\zeta(1)$, again from the Rayleigh-Ritz inequality (Schultz, 1973), we obtain

$$
\int_{0}^{1}|D \zeta|^{2} d z \geqslant \pi^{2} \int_{0}^{1}|\zeta|^{2} d z
$$

Multiplying Eq. (2.4) by $\zeta^{*}$ and integrating over the vertical range of $z$, we get from the real part of the final equation

$$
\begin{aligned}
& \int_{0}^{1}\left(|D \zeta|^{2}+a^{2}|\zeta|^{2}+\sigma_{r}|\zeta|^{2}\right)=\Re \int_{0}^{1} \zeta^{*} D w d z \leqslant\left|\int_{0}^{1} \zeta^{*} D w d z\right| \leqslant \int_{0}^{1}\left|\zeta^{*} D w\right| d z \\
& \quad \leqslant \int_{0}^{1}\left|\zeta^{*}\right||D w| d z \leqslant \int_{0}^{1}|\zeta||D w| d z \leqslant \sqrt{\int_{0}^{1}|\zeta|^{2} d z \sqrt{\int_{0}^{1}|D w|^{2} d z}}
\end{aligned}
$$

(using Schwartz inequality) which implies that

$$
\int_{0}^{1}|D \zeta|^{2} d z \leqslant \sqrt{\int_{0}^{1}|\zeta|^{2} d z \sqrt{\int_{0}^{1}|D w|^{2} d z}}
$$

and thus using inequality (2.19) for the case of rigid boundaries and the result $\int_{0}^{1}|D \zeta|^{2} d z=$ $\pi^{2} \int_{0}^{1}|\zeta|^{2} d z$ for the case of free boundaries (Banerjee et al., 1995), we obtain

$$
\pi^{2} \int_{0}^{1}|\zeta|^{2} d z \leqslant \sqrt{\int_{0}^{1}|\zeta|^{2} d z} \sqrt{\int_{0}^{1}|D w|^{2} d z}
$$


which gives

$$
\sqrt{\int_{0}^{1}|\zeta|^{2} d z} \leqslant \frac{1}{\pi^{2}} \sqrt{\int_{0}^{1}|D w|^{2} d z}
$$

which implies that

$$
\int_{0}^{1}|\zeta|^{2} d z \leqslant \frac{1}{\pi^{4}} \int_{0}^{1}|D w|^{2} d z
$$

Now using inequalities (2.17), (2.18) and (2.21) in Eq. (2.11), we obtain

$$
\begin{aligned}
& {\left[\frac{1}{\operatorname{Pr}}-\left(\frac{R_{1}}{2 \operatorname{Le}_{1}^{2} \pi^{4}}+\frac{R_{2}}{2 \mathrm{Le}_{2}^{2} \pi^{4}}+\ldots+\frac{R_{n-1}}{2 \mathrm{Le}_{n-1}^{2} \pi^{4}}+\frac{\mathrm{Ta}}{\pi^{4}}\right)\right] \int_{0}^{1}|D w|^{2} d z} \\
& +\frac{a^{2}}{\sigma} \int_{0}^{1}|w|^{2} d z+\operatorname{Ra}^{2} \int_{0}^{1}|\theta|^{2} d z<0
\end{aligned}
$$

which clearly implies (for $\sigma_{i} \neq 0$ ) that

$$
\frac{R_{1} \operatorname{Pr}}{2 \mathrm{Le}_{1}^{2} \pi^{4}}+\frac{R_{2} \operatorname{Pr}}{2 \mathrm{Le}_{2}^{2} \pi^{4}}+\ldots+\frac{R_{n-1} \mathrm{Pr}}{2 \mathrm{Le}_{n-1}^{2} \pi^{4}}+\frac{\mathrm{Ta}}{\pi^{4}}>1
$$

Hence if $\frac{R_{1} \operatorname{Pr}}{2 \mathrm{Le}_{1}^{2} \pi^{4}}+\frac{R_{2} \mathrm{Pr}}{2 \mathrm{Le}_{2}^{2} \pi^{4}}+\ldots+\frac{R_{n-1} \mathrm{Pr}}{2 \mathrm{Le}_{n-1}^{2} \pi^{4}}+\frac{\mathrm{Ta}}{\pi^{4}} \leqslant 1$, then we must have $\sigma_{i}=0$.

This proves the theorem.

The essential content of Theorem 1 from the physical point of view is that for the problem of rotatory hydrodynamic multicomponent convection, an arbitrary neutral or unstable mode of the system is definitely non-oscillatory in character and, in particular, "the principle of the exchange of stabilities' is valid if $\frac{R_{1} \operatorname{Pr}}{2 \mathrm{Le}_{1}^{2} \pi^{4}}+\frac{R_{2} \operatorname{Pr}}{2 \mathrm{Le}_{2}^{2} \pi^{4}}+\ldots+\frac{R_{n-1} \mathrm{Pr}}{2 \mathrm{Le}_{n-1}^{2} \pi^{4}}+\frac{\mathrm{Ta}}{\pi^{4}} \leqslant 1$. Further, the above result is uniformly valid for quite general nature of the boundaries.

Special cases: It follows from Theorem 1 that an arbitrary neutral or unstable mode is non oscillatory in character, and in particular PES is valid for:

1. Rayleigh-Benard convection $\left(R_{1}=R_{2}=\ldots=R_{n-1}=\mathrm{Ta}=0\right)$ (Pellew and Southwell, 1940)

2. Rotatory Rayleigh-Benard convection $\left(R_{1}=R_{2}=\ldots=R_{n-1}=0\right)$ if $\mathrm{Ta} / \pi^{4} \leqslant 1$ (Gupta et al., 1986)

3. Rotatory thermohaline convection $\left(R_{1}>0, R_{2}=\ldots=R_{n-1}=0, T_{a}>0\right)$ if $\frac{R_{1} \operatorname{Pr}}{2 \mathrm{Le}_{1}^{2} \pi^{4}}+\frac{\mathrm{Ta}}{\pi^{4}} \leqslant 1$ (Gupta et al., 1986)

4. Thermohaline convection $\left(R_{1}>0, R_{2}=\ldots=R_{n-1}=\mathrm{Ta}=0\right)$ if $\frac{R_{1} \mathrm{Pr}}{2 \mathrm{Le}_{1}^{2} \pi^{4}} \leqslant 1$ (Gupta et al., 1986)

5. Rotatory hydrodynamic triply diffusive convection $\left(R_{1}>0, R_{2}>0, R_{3}=\ldots=R_{n-1}=0\right.$, $\mathrm{Ta}>0)$ if $\frac{R_{1} \mathrm{Pr}}{2 \mathrm{Le}_{1}^{2} \pi^{4}}+\frac{R_{2} \mathrm{Pr}}{2 \mathrm{Le}_{2}^{2} \pi^{4}}+\frac{\mathrm{Ta}}{\pi^{4}} \leqslant 1$ (Prakash et al., 2014b)

6. Triply diffusive convection $\left(R_{1}>0, R_{2}>0, R_{3}=\ldots=R_{n-1}=\mathrm{Ta}=0\right)$ if $\frac{R_{1} \mathrm{Pr}}{2 \mathrm{Le}_{1}^{2} \pi^{4}}+\frac{R_{2} \mathrm{Pr}}{2 \mathrm{Le}_{2}^{2} \pi^{4}} \leqslant 1$ (Prakash et al., 2014a) 
Proceeding in this manner, we can obtain conditions for stationary convection for all configurations with $3,4, \ldots, n-1$ concentration components, respectively.

Since the complement of the above result implies the occurrence of oscillatory motions, thus it is important to derive the bounds for the complex growth rate of oscillatory motions. We prove the following theorem in this direction.

Theorem 2: If $R_{a}>0, R_{1}>0, R_{2}>0, \ldots, R_{n-1}>0$, Ta $>0, \sigma_{r} \geqslant 0$ and $\sigma_{i} \neq 0$, then a necessary condition for the existence of a nontrivial solution $\left(w, \theta, \phi_{1}, \phi_{2}, \ldots, \phi_{n-1}, \zeta, \sigma\right)$ to Eqs. (2.3) and (2.) together with boundary conditions (2.5)-(2.7) is that

$$
|\sigma|<\max \left\{\sqrt{\left(R_{1}+R_{2}+\ldots+R_{n-1}\right) \operatorname{Pr}}, \sqrt{\operatorname{Ta}} \operatorname{Pr}\right\}
$$

Proof: Rewriting equation (2.11) for ready reference, we have

$$
\begin{aligned}
& \frac{1}{\operatorname{Pr}} \int_{0}^{1}\left(|D w|^{2}+a^{2}|w|^{2}\right) d z=-\operatorname{Ra}^{2} \int_{0}^{1}|\theta|^{2} d z+R_{1} a^{2} \int_{0}^{1}\left|\phi_{1}\right|^{2} d z \\
& \quad+R_{2} a^{2} \int_{0}^{1}\left|\phi_{2}\right|^{2} d z+\ldots+R_{n-1} a^{2} \int_{0}^{1}\left|\phi_{n-1}\right|^{2} d z+\frac{\operatorname{Ta}}{\operatorname{Pr}} \int_{0}^{1}|\zeta|^{2} d z
\end{aligned}
$$

Now since $\sigma_{r} \geqslant 0$, it follows from Eq. (2.13) that

$$
\int_{0}^{1}\left|\phi_{1}\right|^{2} d z \leqslant \frac{1}{|\sigma|^{2}} \int_{0}^{1}|w|^{2} d z
$$

Similarly, from Eqs. $(2.3)_{4}$ and $(2.3)_{6}$, by adopting the same procedure, we get

$$
\begin{aligned}
& \int_{0}^{1}\left|\phi_{2}\right|^{2} d z \leqslant \frac{1}{|\sigma|^{2}} \int_{0}^{1}|w|^{2} d z \\
& \vdots \\
& \int_{0}^{1}\left|\phi_{n-1}\right|^{2} d z \leqslant \frac{1}{|\sigma|^{2}} \int_{0}^{1}|w|^{2} d z
\end{aligned}
$$

respectively.

Multiply Eq. (2.4) by its complex conjugate, integrating the resulting equation by parts for an appropriate number of times and using boundary conditions (2.5)-(2.7), we have

$$
\begin{aligned}
& \int_{0}^{1}\left(\left|D^{2} \zeta\right|^{2}+2 a^{2}|D \zeta|^{2}+a^{4}|\zeta|^{2}\right) d z+\frac{2 \sigma_{r}}{\operatorname{Pr}} \int_{0}^{1}\left(|D \zeta|^{2}+a^{2}|\zeta|^{2}\right) d z \\
& +\frac{|\sigma|^{2}}{\operatorname{Pr}^{2}} \int_{0}^{1}|\zeta|^{2} d z=\int_{0}^{1}|D w|^{2} d z
\end{aligned}
$$

Since, $\sigma_{r} \geqslant 0$, it follows from Eq. (2.26) that

$$
\int_{0}^{1}|\zeta|^{2} d z \leqslant \frac{\operatorname{Pr}^{2}}{|\sigma|^{2}} \int_{0}^{1}|D w|^{2} d z
$$


Now making use of inequalities (2.24), (2.25) and (2.27), in Eq. (2.11), we have

$$
\begin{aligned}
& \frac{1}{\operatorname{Pr}}\left(1-\frac{\operatorname{TaPr}^{2}}{|\sigma|^{2}}\right) \int_{0}^{1}|D w|^{2} d z+\frac{a^{2}}{\operatorname{Pr}}\left[1-\frac{\left(R_{1}+R_{2}+\ldots+R_{n-1}\right) \operatorname{Pr}}{|\sigma|^{2}}\right] \int_{0}^{1}|w|^{2} d z \\
& \quad+\operatorname{Ra} a^{2} \int_{0}^{1}|\theta|^{2} d z<0
\end{aligned}
$$

which clearly implies that

$$
|\sigma|<\max \left\{\sqrt{\left(R_{1}+R_{2}+\ldots+R_{n-1}\right) \operatorname{Pr}}, \sqrt{\operatorname{Ta}} \operatorname{Pr}\right\}
$$

This establishes the desired result.

The above theorem may be stated in an equivalent form as: the complex growth rate of an arbitrary, neutral or unstable oscillatory perturbation of growing amplitude in a rotatory hydrodynamic multicomponent fluid layer heated from below must lie inside a semicircle in the right half of the $\left(p_{r}, p_{i}\right)$-plane whose centre is at the origin and radius equals $\max \left\{\sqrt{\left(R_{1}+R_{2}+\ldots+R_{n-1}\right) \operatorname{Pr}}, \sqrt{\mathrm{Ta} P r}\right\}$. Further, it is proved that this result is uniformly valid for quite general nature of the bounding surfaces.

Special cases: The following results may be obtained from Theorem 2 as special cases:

1. For rotatory Rayleigh-Benard convection $\left(R_{1}=0=R_{2}=\ldots=R_{n-1}=0\right.$, Ta $\left.>0\right)$

$$
|\sigma|<\operatorname{TaPr}
$$

(Banerjee et al., 1981)

2. For thermohaline convection $\left(R_{1}>0, R_{2}=\ldots=R_{n-1}=\mathrm{Ta}=0\right)$

$$
|\sigma|<\sqrt{R_{1} \operatorname{Pr}}
$$

(Banerjee et al., 1981)

3. For rotatory Thermohaline convection of the Veronis type (Turner, 1985) $\left(R_{1}>0\right.$, $\left.R_{2}=\ldots=R_{n-1}=0, \mathrm{Ta}>0\right)$

$$
|\sigma|<\max \left\{\sqrt{R_{1} \operatorname{Pr}}, \sqrt{\operatorname{Ta}} \operatorname{Pr}\right\}
$$

(Gupta et al., 1983)

4. For triply diffusive convection $\left(R_{1}>0, R_{2}>0, R_{3}=\ldots=R_{n-1}=\mathrm{Ta}=0\right)$

$$
|\sigma|<\sqrt{\left(R_{1}+R_{2}\right) \operatorname{Pr}}
$$

(Prakash et al., 2014c)

Proceeding in this manner, we can obtain bounds for the complex growth rate for all configurations with $3,4, \ldots, n-1$ concentration components, respectively. 


\section{Conclusions}

The present analysis generalizes the previous published results for rotatory hydrodynamic singly, doubly and triply diffusive convection. The mathematical analysis carried out here yields a sufficient condition for the validity of the principle of the exchange of stabilities in rotatory hydrodynamic multicomponent convection. Since the complement of this condition implies the occurrence of oscillatory motions, the bounds for the complex growth rate are also obtained as the second problem. It is further proved that the results obtained herein are uniformly applicable for quite general nature of bounding surfaces.

\section{Acknowledgment}

The authors would like to thank the learned reviewer for his valuable comments, which helped the authors to bring the manuscript in the present form. First author (J. Prakash) also acknowledges the recently awarded financial assistance by UGC, New Delhi in the form of major research project (Grant number, 43-420/2014(SR)).

\section{References}

1. Banerjee M.B., Katoch D.C., Dube G.S., Banerjee K., 1981, Bounds for growth rate of perturbation in thermohaline convection, Proceedings of the Royal Society of London, A, 378, 301-304

2. Banerjee M.B., Shandil R.G., Lal P., Kanwar V., 1995, A mathematical theorem in rotatory thermohaline convection, Journal of Mathematics Analysis and Applied, 189, 351-361

3. Brandt A., Fernando H.J.S., 1996, Double diffusive convection, American Geophysical Union, Washington, DC

4. Griffiths R.W., 1979a, A note on the formation of salt finger and diffusive interfaces in three component systems, International Journal of Heat and Mass Transfer, 22, 1687-1693

5. GRIFFiths R.W., 1979b, The influence of a third diffusing component upon the onset of convection, Journal of Fluid Mechanics, 92, 659-670

6. Gupta J.R., Sood S.K., Bhardwaj U.D., 1986, On the characterization of non-oscillatory motions in a rotatory hydromagnetic thermohaline convection, Indian Journal of Pure and Applied Mathematics, 17, 1, 100-107

7. Gupta J.R., Sood S.K., Shandil R.G., Banerjee M.B., Banerjee K., 1983, Bounds for the growth of a perturbation in some double-diffusive convection problems, Journal of Australian Mathematics Society, Ser. B, 25, 276-285

8. Kellner M., Tilgner A., 2014, Transition to finger convection in double diffusive convection, Physics of Fluids, 26, 094103

9. Lopez A.R., Romero L.A., Pearlstein A.J., 1990, Effect of rigid boundaries on the onset of convective instability in a triply diffusive fluid layer, Physics of Fluids A, 2, 6, 897-902

10. Nield D.A., Kuznetsov A.V., 2011, The onset of double-diffusive convection in a nano fluid layer, International Journal Heat Fluid Flow, 32, 4, 771-776

11. Pearlstein A.J., Harris R.M., Terrones G., 1989, The onset of convective instability in a triply diffusive fluid layer, Journal of Fluid Mechanics, 202, 443-465

12. Pellew A., Southwell R.V., 1940, On the maintained convective motion in a fluid heated from below, Proceedings of the Royal Society of London, A, 176, 312-343

13. Poulikakos D., 1985, The effect of a third diffusing component on the onset of convection in a horizontal porous layer, Physics of Fluids, 28, 10, 3172-3174 
14. Prakash J., Bala R., VAid K., 2014a, On the characterization of nonoscillatory motions in triply diffusive convection, International Journal of Fluid Mechanics Research, 41, 5, 409-416

15. Prakash J., Bala R., VAid K., 2014b, On the principle of the exchange of stabilities in rotatory triply diffusive convection, Proceeding National Academy Science, Physical Sciences, India, 84, 3, 433-439

16. Prakash J., BAla R., VAID K., 2014c, Upper limits to the complex growth rates in triply diffusive Convection, Proceeding Indian National Science Academy, 80, 1, 115-122

17. Prakash J., Bala R., Vaid K., 2015, On arresting the complex growth rates in Rotatory Triply Diffusive convection, communicated for publication

18. Radko T., 2013, Double-Diffusive Convection, Cambridge university Press

19. Rionero S., 2013a, Multicomponent diffusive-convective fluid motions in porous layers ultimately boundedness, absence of subcritical instabilities, and global nonlinear stability for any number of salts, Physics of Fluids, 25, 054104, 1-23

20. Rionero S., 2013b, Triple diffusive convection in porous media, Acta Mechanics, 224, 447-458

21. Rionero S., 2014, Onset of convection in rotating porous layers via a new approach, Discrete and Continuous Dynamical Systems, Ser. B, 19, 7, 2279-2296

22. Ryzhkov I.I., 2013, Long-wave instability of a plane multicomponent mixture layer with the soret effect, Fluid Dynamics, 4, 48, 477-490

23. Ryzhkov I.I., Shevtsova V.M., 2007, On thermal diffusion and convection in multicomponent mixtures with application to the thermogravitational column, Physics of Fluids, 19, 027101, 1-17

24. Ryzhkov I.I., Shevtsova V.M., 2009, Long wave instability of a multicomponent fluid layer with the soret effect, Physics of Fluids, 21, 014102, 1-14

25. Schmitt R.W., 2011, Thermohaline convection at density ratios below one: A new regime for salt fingers, Journal of Marine Research, 69, 779-795

26. Schultz M.H., 1973, Spline Analysis, Prentice-Hall Inc. Englewood Cliffs NJ

27. Sekar R., Raju K., Vasanthakumari R., 2013, A linear analytical study of Soret-driven Ferro thermohaline convection in an anisotropic porous medium, Journal of Magnetism and Magnetic Materials, 331, 122-128

28. Shivakumara I.S., Naveen Kumar S.B., 2014, Linear and weakly nonlinear triple diffusive convection in a couple stress fluid layer, International Journal of Heat and Mass Transfer, 68, 542-553

29. Terrones G., 1993, Cross-diffusion effects on the stability criteria in a triply diffusive system, Physics of Fluids A, 5, 9, 2172-2182

30. Terrones G., Pearlstein A.J., 1989, The onset of convection in a multicomponent fluid layer, Physics of Fluids A, 1, 5, 845-853

31. Turner J.S., 1973, Buoyancy Effects in Fluids, Cambridge University Press

32. Turner J.S., 1974, Double diffusive phenomenon, Annual Review of Fluid Mechanics, 6, 37-56

33. Turner J.S., 1985, Multicomponent convection, Annual Review of Fluid Mechanics, 17, 11-44 